\title{
Multiple solutions for a class of superquadratic fractional Hamiltonian systems
}

\author{
Mohsen Timoumi ${ }^{\mathrm{a}^{*}}$ \\ ${ }^{a}$ Department of Mathematics, Faculty of Sciences, Monastir University, Monastir, Tunisia \\ *Corresponding author E-mail: m_timoumi@yahoo.com
}

\begin{abstract}
Article Info
Keywords: Fractional Hamiltonian systems, Variational methods, Symmetric Mountain Pass Theorem.

2010 AMS: 34C37, 35A15, 35B38

Received: 1 February 2018

Accepted: 3 April 2018

Available online: 30 September 2018
\end{abstract}

\begin{abstract}
In this paper, we are concerned with the existence of solutions for a class of fractional Hamiltonian systems

$$
\left\{\begin{array}{l}
{ }_{t} D_{\infty}^{\alpha}\left({ }_{-\infty} D_{t}^{\alpha} u\right)(t)+L(t) u(t)=\nabla W(t, u(t)), t \in \mathbb{R} \\
u \in H^{\alpha}\left(\mathbb{R}, \mathbb{R}^{N}\right)
\end{array}\right.
$$

where ${ }_{t} D_{\infty}^{\alpha}$ and ${ }_{-\infty} D_{t}^{\alpha}$ are the Liouville-Weyl fractional derivatives of order $\frac{1}{2}<\alpha<1, L \in$ $C\left(\mathbb{R}, \mathbb{R}^{N^{2}}\right)$ is a symmetric matrix-valued function and $W(t, x) \in C^{1}\left(\mathbb{R} \times \mathbb{R}^{N}, \mathbb{R}\right)$. Applying a Symmetric Mountain Pass Theorem, we prove the existence of infinitely many solutions for (1) when $L$ is not required to be either uniformly positive definite or coercive and $W(t, x)$ satisfies some weaker superquadratic conditions at infinity in the second variable but does not satisfy the well-known Ambrosetti-Rabinowitz superquadratic growth condition.
\end{abstract}

\section{Introduction.}

Fractional differential equations both ordinary and partial ones have attracted extensive attentions because of their applications in mathematical modeling of processes in physics, mechanics, control theory, viscoelasticity, electro chemistry, bioengineering, economics and others. Therefore, the theory of fractional differential equations is an area intensively developed during the last decades [1], [11]. The monographs [13], [17], [18] enclose a review of methods of solving fractional differential equations, which are an extension of procedures from differential equations theory.

Recently, many results were obtained dealing with the existence and multiplicity of solutions of nonlinear fractional differential equations by using techniques of Nonlinear Analysis, such as fixed point theory [5], [25], topological degree theory [6], [19], comparison methods [14], [24], and so on.

It should be noted that critical point theory and variational methods serve as effective tools in the study of integer-order differential equations. The underlying idea in this approach rest on finding critical points for suitable energy functional defined on an appropriate function space. During the last three decades, the critical point theory has been developed into a wonderful tool for investigating the existence criteria for the solutions of differential equations with variational structures, for example see [15], [19] and the references cited therein.

Motivated by the classical works in [15], [19], for the first time, the author [10] showed that critical point theory and variational methods are an effective approach to tackle the existence of solutions for the following fractional boundary value problem

$$
\left\{\begin{array}{l}
{ }_{t} D_{T}^{\alpha}\left({ }_{0} D_{t}^{\alpha} u\right)(t)=\nabla W(t, u(t)), t \in[0, T] \\
u(0)=u(T)
\end{array}\right.
$$

where $\frac{1}{2}<\alpha<1, W \in C^{1}\left(\mathbb{R} \times \mathbb{R}^{N}, \mathbb{R}\right)$ with derivative $\nabla W(t, x)=\frac{\partial W}{\partial x}(t, x)$, and obtained the existence of at least one nontrivial solution. Inspired by this work, Torres [20] considered the following fractional Hamiltonian system

$$
\mathscr{F} \mathscr{H} \mathscr{S} \quad\left\{\begin{array}{l}
{ }_{t} D_{\infty}^{\alpha}\left({ }_{-} D_{t}^{\alpha} u\right)(t)+L(t) u(t)=\nabla W(t, u(t)), t \in \mathbb{R} \\
u \in H^{\alpha}\left(\mathbb{R}, \mathbb{R}^{N}\right),
\end{array}\right.
$$


where $\frac{1}{2}<\alpha<1, W(t, x)$ is as above and $L$ satisfies

(1.1) $L \in C\left(\mathbb{R}, \mathbb{R}^{N^{2}}\right)$ is a positive definite symmetric matrix-valued function, and there exists an $l \in C\left(\mathbb{R}, \mathbb{R}_{+}^{*}\right)$ such that $l(t) \longrightarrow+\infty$ as $|t| \longrightarrow \infty$ and

$$
L(t) x \cdot x \geq l(t)|x|^{2}, \forall(t, x) \in \mathbb{R} \times \mathbb{R}^{N} .
$$

Assuming that $W \in C^{1}\left(\mathbb{R} \times \mathbb{R}^{N}, \mathbb{R}\right)$ satisfies the well-known Ambrosetti-Rabinowitz superquadratic condition $(\mathscr{A} \mathscr{R})$ and some other suitable conditions, the author [20] showed that the fractional Hamiltonian system ( $\mathscr{F} \mathscr{H} \mathscr{S}$ ) possesses at least one nontrivial solution using the Mountain Pass Theorem. Since then, the existence and multiplicity of solutions of problem $(\mathscr{F} \mathscr{H} \mathscr{S})$ via critical point theory have been investigated in many papers [3,4,7,8,16,20-23,25-28].

Recently, Mèndez and Torres [16] proved the existence of multiple solutions for the fractional Hamiltonian system ( $\mathscr{F} \mathscr{H} \mathscr{S}$ ) when the potential $W$ satisfies some subquadratic conditions at infinity and the matrix-valued function $L$ satisfies the following noncoercive conditions $\left(L_{1}\right) L(t)$ is a positive definite symmetric matrix for all $t \in \mathbb{R}$ and there exists an $l \in C(\mathbb{R}, \mathbb{R})$ such that

$$
\inf _{t \in \mathbb{R}} l(t)>0 \text { and } L(t) x . x \geq l(t)|x|^{2}, \forall(t, x) \in \mathbb{R} \times \mathbb{R}^{N} ;
$$

$\left(L_{2}\right)$ There exists a constant $r_{0}>0$ such that

$$
\lim _{|s| \longrightarrow \infty} \operatorname{meas}\left(\{t \in] s-r_{0}, s+r_{0}\left[/ L(t)<b I_{N}\right\}\right)=0, \forall b>0,
$$

where meas denotes the Lebesgue's measure on $\mathbb{R}$. The above conditions on $L$ guarantee the compactness of Sobolev embedding. Besides, in all the above mentioned papers, the potential $W$ is required to be subquadratic or to satisfy the Ambrosetti-Rabinowitz superquadratic condition $(\mathscr{A} \mathscr{R})$ at infinity.

The aim of this paper is to study the existence of infinitely many solutions for ( $\mathscr{F} \mathscr{H} \mathscr{S}$ ), when the function $L$ is unnecessarily positive definite or coercive, and the potential $W$ satisfies some superquadratic conditions at infinity, weaker than the $(\mathscr{A} \mathscr{R})-$ condition. More precisely, let $W \in C^{1}\left(\mathbb{R} \times \mathbb{R}^{N}, \mathbb{R}\right)$ be such that for all $r>0, \nabla W$ is bounded in $\mathbb{R} \times B_{r}(0)$, we make the following hypotheses:

$(L)$ The smallest eigenvalue of $L(t)$ is bounded from below;

$\left(W_{1}\right) \quad W(t, 0)=0$ and $\nabla W(t, x)=o(|x|)$, as $|x| \longrightarrow 0$ uniformly on $t \in \mathbb{R} ;$

$$
\lim _{|x| \rightarrow+\infty} \frac{|W(t, x)|}{|x|^{2}}=+\infty, \forall t \in \mathbb{R}
$$

and

$$
W(t, x) \geq 0, \forall(t, x) \in \mathbb{R} \times \mathbb{R}^{N} \text { with }|x| \geq R_{0} ; W(t,-x)=W(t, x), \forall(t, x) \in \mathbb{R} \times \mathbb{R}^{N} ;
$$

$\left(W_{4}\right)$ There exist $g \in L^{1}(\mathbb{R})$ and constants $b_{0}, c_{0}>0$ and $\left.v \in\right] 0,2[$ such that

$$
\widehat{W}(t, x)=\frac{1}{2} \nabla W(t, x) \cdot x-W(t, x) \geq\left\{\begin{array}{l}
g(t), \forall t \in \mathbb{R},|x| \leq R_{0} \\
b_{0}|x|^{v}, \forall t \in \mathbb{R},|x| \geq R_{0}
\end{array}\right.
$$

$|W(t, x)| \leq c_{0}|x|^{2-v} \widehat{W}(t, x), \forall(t, x) \in \mathbb{R} \times \mathbb{R}^{N}$ with $|x| \geq R_{0} ;$

$\left(W_{5}\right)$ There exist constants $\mu>2$ and $\rho_{0}>0$ such that

$$
\mu W(t, x) \leq \nabla W(t, x) \cdot x+\rho_{0}|x|^{2}, \forall(t, x) \in \mathbb{R} \times \mathbb{R}^{N} .
$$

Our main results read as follows.

Theorem 1.1. Assume that $(L),\left(L_{2}\right)$ and $\left(W_{1}\right)-\left(W_{4}\right)$ are satisfied. Then $(\mathscr{F} \mathscr{H} \mathscr{S})$ possesses infinitely many nontrivial solutions.

Theorem 1.2. Assume that $(L),\left(L_{2}\right),\left(W_{1}\right)-\left(W_{3}\right)$ and $\left(W_{5}\right)$ are satisfied. Then $(\mathscr{F} \mathscr{H} \mathscr{S})$ possesses infinitely many nontrivial solutions.

Remark 1.3. $\quad$ 1. In our results, $L(t)$ is unnecessarily required to be either uniformly positive definite or coercive. For example $L(t)=\left(t^{2} \sin ^{2} t-1\right) I_{N}$, where $I_{N}$ is the identity matrix, satisfies $(L)$ and $\left(L_{2}\right)$, but it does satisfy neither Theorem 1.1 nor Theorem 1.2.

2. Let $W(t, x)=a(t)|x|^{2} \ln \left(\frac{1}{2}+|x|\right)$, where a is a continuous bounded function with positive lower bound. Then an easy computation shows that $W$ satisfies the superquadratic conditions $\left(W_{1}\right)-\left(W_{4}\right)$. However, $W$ does not satisfy the $(\mathscr{A} \mathscr{R})-$ condition.

The remainder of this paper is organized as follows. In Section 2, some preliminary results are presented. Section 3 is devoted to the proofs of our results.

\section{Preliminaries}

In this Section, for the reader's convenience, first we will recall some facts about the fractional calculus on the whole real axis. On the other hand, we will give some preliminaries lemmas for using in the sequel. 


\subsection{Liouville-Weyl fractional calculus}

The Liouville-Weyl fractional integrals of order $0<\alpha<1$ on the whole axis $\mathbb{R}$ are defined as (see [12], [13], [18])

$$
{ }_{-\infty} I_{t}^{\alpha} u(t)=\frac{1}{\Gamma(\alpha)} \int_{-\infty}^{t}(t-x)^{\alpha-1} u(x) d x,
$$

and

$$
{ }_{t} I_{\infty}^{\alpha} u(t)=\frac{1}{\Gamma(\alpha)} \int_{t}^{\infty}(x-t)^{\alpha-1} u(x) d x
$$

The Liouville-Weyl fractional derivatives of order $0<\alpha<1$ on the whole axis $\mathbb{R}$ are defined as the left-inverse operators of the corresponding Liouville-Weyl fractional integrals (see [12], [13], [18])

$$
{ }_{\infty} D_{t}^{\alpha} u(t)=\frac{d}{d t}\left({ }_{-\infty} I_{t}^{1-\alpha} u\right)(t),
$$

and

$$
{ }_{t} D_{\infty}^{\alpha} u(t)=-\frac{d}{d t}\left({ }_{t} I_{\infty}^{1-\alpha} u\right)(t) .
$$

The definitions of 2.1 and 3.2 may be written in an alternative form as follows

$$
{ }_{-\infty} D_{t}^{\alpha} u(t)=\frac{1}{\Gamma(1-\alpha)} \int_{0}^{\infty} \frac{u(t)-u(t-x)}{x^{\alpha+1}} d x,
$$

and

$$
{ }_{t} D_{\infty}^{\alpha} u(t)=\frac{1}{\Gamma(1-\alpha)} \int_{0}^{\infty} \frac{u(t)-u(t+x)}{x^{\alpha+1}} d x .
$$

We establish the Fourier transform properties of the fractional integral and fractional differential operators. Recall that the Fourier transform $\widehat{u}$ of $u$ is defined by

$$
\widehat{u}(s)=\int_{-\infty}^{\infty} e^{-i s t} u(t) d t
$$

Let $u$ be defined on $\mathbb{R}$. Then the Fourier transform of the Liouville-Weyl integrals and differential operators satisfies (see [12,13])

$$
\begin{aligned}
& \widehat{{ }_{-\infty} I_{t}^{\alpha} u}(s)=(i s)^{-\alpha} \widehat{u}(s), \\
& \widehat{{ }_{t}{ }_{\infty}^{\alpha} u}(s)=(-i s)^{-\alpha} \widehat{u}(s), \\
& \widehat{{ }_{\infty} D_{t}^{\alpha}} u(s)=(i s)^{\alpha} \widehat{u}(s), \\
& { }_{{ }_{t}{ }^{\alpha} u} u(s)=(-i s)^{\alpha} \widehat{u}(s) .
\end{aligned}
$$

Next, we present some properties for Liouville-Weyl fractional integrals and derivatives on the real axis, which were proved in [12]. Denote by $L^{p}\left(\mathbb{R}, \mathbb{R}^{N}\right)(1 \leq p<\infty)$, the Banach spaces of functions on $\mathbb{R}$ with values in $\mathbb{R}^{N}$ under the norms

$$
\|u\|_{L^{p}}=\left(\int_{\mathbb{R}}|u(t)|^{p} d t\right)^{\frac{1}{p}}
$$

and $L^{\infty}\left(\mathbb{R}, \mathbb{R}^{N}\right)$ the Banach space of essentially bounded functions from $\mathbb{R}$ into $\mathbb{R}^{N}$ equipped with the norm

$$
\|u\|_{\infty}=\operatorname{esssup}\{|u(t)| / t \in \mathbb{R}\} .
$$

\subsection{Fractional derivative spaces}

In order to establish the variational structure which enables us to reduce the existence of solutions of $(\mathscr{F} \mathscr{H} \mathscr{S})$ to find critical points of the corresponding functional, it is necessary to construct the appropriate functional spaces.

For $\alpha>0$, define the semi-norm

$$
|u|_{I_{-\infty}^{\alpha}}=\|\|_{-\infty} D_{t}^{\alpha} u \|_{L^{2}}
$$

and the norm

$$
\|u\|_{I_{-\infty}^{\alpha}}=\left(\|u\|_{L^{2}}+|u|_{I_{-\infty}^{\alpha}}^{2}\right)^{\frac{1}{2}}
$$

and let

$$
I_{-\infty}^{\alpha}=\overline{C_{0}^{\infty}\left(\mathbb{R}, \mathbb{R}^{N}\right)}\|\cdot\| \|_{I_{-\infty}^{\alpha}}
$$

where $C_{0}^{\infty}\left(\mathbb{R}, \mathbb{R}^{N}\right)$ denotes the space of infinitely differentiable functions from $\mathbb{R}$ into $\mathbb{R}^{N}$ with vanishing property at infinity. Now, we can define the fractional Sobolev space $H^{\alpha}\left(\mathbb{R}, \mathbb{R}^{N}\right)$ in terms of the Fourier transform. Choose $0<\alpha<1$, define the semi-norm

$$
|u|_{\alpha}=\left\||s|^{\alpha} \widehat{u}\right\|_{L^{2}}
$$


and the norm

$$
\|u\|_{\alpha}=\left(\|u\|_{L^{2}}+|u|_{\alpha}^{2}\right)^{\frac{1}{2}}
$$

and let

$$
H^{\alpha}\left(\mathbb{R}, \mathbb{R}^{N}\right)=\overline{C_{0}^{\infty}\left(\mathbb{R}, \mathbb{R}^{N}\right)}\|\cdot\|_{\alpha}
$$

Moreover, we note that a function $u \in L^{2}\left(\mathbb{R}, \mathbb{R}^{N}\right)$ belongs to $I_{-\infty}^{\alpha}$ if and only if

$$
|s|^{\alpha} \widehat{u} \in L^{2}\left(\mathbb{R}, \mathbb{R}^{N}\right)
$$

Especially, we have

$$
|u|_{I_{-\infty}^{\alpha}}=\left\||s|^{\alpha} \widehat{u}\right\|_{L^{2}} .
$$

Therefore, $I_{-\infty}^{\alpha}$ and $H^{\alpha}\left(\mathbb{R}, \mathbb{R}^{N}\right)$ are equivalent with equivalent semi-norms and norms. Analogous to $I_{-\infty}^{\alpha}$, we introduce $I_{\infty}^{\alpha}$. Define the semi-norm

$$
|u|_{I_{\infty}^{\alpha}}=\left\|{ }_{t} D_{\infty}^{\alpha} u\right\|_{L^{2}}
$$

and the norm

$$
\|u\|_{I_{\infty}^{\alpha}}=\left(\|u\|_{L^{2}}+|u|_{I_{\infty}^{\alpha}}^{2}\right)^{\frac{1}{2}}
$$

and let

$$
I_{\infty}^{\alpha}=\overline{C_{0}^{\infty}\left(\mathbb{R}, \mathbb{R}^{N}\right)}\|\cdot\|_{I_{\infty}^{\alpha}}
$$

Then $I_{-\infty}^{\alpha}$ and $I_{\infty}^{\alpha}$ are equivalent with equivalent semi-norms and norms.

Let $C\left(\mathbb{R}, \mathbb{R}^{N}\right)$ denotes the space of continuous functions from $\mathbb{R}$ into $\mathbb{R}^{N}$. Then we obtain the following Sobolev lemma.

Lemma 2.1. [[21], Theorem 2.1]. If $\alpha>\frac{1}{2}$, then $H^{\alpha}\left(\mathbb{R}, \mathbb{R}^{N}\right) \subset C\left(\mathbb{R}, \mathbb{R}^{N}\right)$, and there exists a constant $C=C_{\alpha}$ such that

$$
\|u\|_{\infty}=\sup _{t \in \mathbb{R}}|u(t)| \leq C_{\alpha}\|u\|_{\alpha}, \forall u \in H^{\alpha}\left(\mathbb{R}, \mathbb{R}^{N}\right) .
$$

Remark 2.2. From Lemma 2.1, we know that if $u \in H^{\alpha}\left(\mathbb{R}, \mathbb{R}^{N}\right)$ with $\frac{1}{2}<\alpha<1$, then $u \in L^{p}\left(\mathbb{R}, \mathbb{R}^{N}\right)$ for all $\left.p \in\right] 2, \infty[$, because

$$
\int_{\mathbb{R}}|u(t)|^{p} d t \leq\|u\|_{\infty}^{p-2}\|u\|_{L^{2}}^{2} .
$$

In this section, we assume the $L$ satisfies the following condition

$$
\left(L_{0}\right) \quad L(t) x . x \geq|x|^{2}, \forall(t, x) \in \mathbb{R} \times \mathbb{R}^{N}
$$

and we introduce the following fractional space

$$
X^{\alpha}=\left\{u \in H^{\alpha}\left(\mathbb{R}, \mathbb{R}^{N}\right) / \int_{\mathbb{R}} L(t) u(t) \cdot u(t) d t<\infty\right\} .
$$

Then $X^{\alpha}$ is a Hilbert space with the inner product

$$
<u, v>_{X^{\alpha}}=\int_{\mathbb{R}}\left[{ }_{-\infty} D_{t}^{\alpha} u(t) \cdot-_{\infty} D_{t}^{\alpha} v(t)+L(t) u(t) \cdot v(t)\right] d t
$$

and the corresponding norm

$$
\|u\|_{X^{\alpha}}^{2}=<u, u>_{X^{\alpha}}
$$

It is easy to see that $X^{\alpha}$ is continuously embedded in $H^{\alpha}\left(\mathbb{R}, \mathbb{R}^{N}\right)$ and in $L^{2}\left(\mathbb{R}, \mathbb{R}^{N}\right)$. In fact, for $u \in X^{\alpha}$, we have

$$
\|u\|_{X^{\alpha}}^{2}=\int_{\mathbb{R}}\left[\left.\left.\right|_{-\infty} D_{t}^{\alpha} u(t)\right|^{2}+L(t) u(t) \cdot u(t)\right] d t \geq \int_{\mathbb{R}}\left[\left.\left.\right|_{-\infty} D_{t}^{\alpha} u(t)\right|^{2}|u(t)|^{2}\right] d t=\|u\|_{H^{\alpha}}^{2} \geq\|u\|_{L^{2}}^{2} .
$$

For $p \in] 2, \infty[$, we have by Remark 2.2

$$
\|u\|_{L^{p}}^{p}=\int_{\mathbb{R}}|u(t)|^{p} d t \leq\|u\|_{\infty}^{p-2}\|u\|_{L^{2}}^{2} \leq C_{\alpha}^{p-2}\|u\|_{X^{\alpha}}^{p} .
$$

Hence for all $p \in[1, \infty]$, there exists a constant $\eta_{p}>0$ such that

$$
\|u\|_{L^{p}}^{p} \leq \eta_{p}\|u\|_{X^{\alpha}}^{p}
$$

The main difficulty in dealing with the existence of infinitely many solutions for $(\mathscr{F} \mathscr{H} \mathscr{S})$ is the lack of compactness of the Sobolev embedding. To overcome this difficulty under the assumptions of Theorems 1.1 and 1.2, we employ the following compact embedding lemma. 
Lemma 2.3. [16] Assume $\left(L_{0}\right)$ and $\left(L_{2}\right)$ are satisfied. Then $X^{\alpha}$ is compactly embedded in $L^{2}\left(\mathbb{R}, \mathbb{R}^{N}\right)$.

Remark 2.4. From Remark 2.2 and Lemma 2.3 , it is easy to verify that the embedding of $X^{\alpha}$ in $L^{p}\left(\mathbb{R}, \mathbb{R}^{N}\right)$ is also compact for $\left.p \in\right] 2, \infty[$.

To study the critical points of the variational functional associated with $(\mathscr{F} \mathscr{H} \mathscr{S})$, we need to recall the Symmetric Mountain Pass Theorem [19].

Definition 2.5. Let $X$ be a Banach space with the norm $\|$.$\| , we say that \Phi \in C^{1}(X, \mathbb{R})$ satisfies the a) $(P S)_{c}$-condition, $c \in \mathbb{R}$, if any sequence $\left(u_{n}\right) \subset X$ satisfying

$$
\Phi\left(u_{n}\right) \longrightarrow c \text { and } \Phi^{\prime}\left(u_{n}\right) \longrightarrow 0 \text { as } n \longrightarrow \infty
$$

possesses a convergent subsequence,

b) $(C)_{c}$-condition, $c \in \mathbb{R}$, if any sequence $\left(u_{n}\right) \subset X$ satisfying

$$
\Phi\left(u_{n}\right) \longrightarrow c \text { and }\left\|\Phi^{\prime}\left(u_{n}\right)\right\|\left(1+\left\|u_{n}\right\|\right) \longrightarrow 0 \text { as } n \longrightarrow \infty
$$

possesses a convergent subsequence.

Lemma 2.6. Let $X$ be an infinite dimensional Banach space, $X=Y \oplus Z$, where $Y$ is finite dimensional space. Suppose that $\Phi \in C^{1}(X, \mathbb{R})$ satisfies the Palais-Smale condition and

(a) $\Phi(0)=0, \Phi(-u)=\Phi(u), \forall u \in X$

(b) Thereexistconstants $\rho, \alpha>0$ suchthat $\Phi_{\mid \partial B_{\rho} \cap Z} \geq \alpha$;

(c) Foranyfinitedimensionalsubspace $\widetilde{E} \subset X$, thereis $R=R(\widetilde{E})>0$ suchthat $\Phi(u) \leq 0$ on $\widetilde{E} \backslash B_{R}$, where $B_{R}=\{u \in X /\|u\|<R\}$.

Then $\Phi$ possesses an unbounded sequence of critical values.

Remark 2.7. As shown in [2], a deformation lemma can be proved with $(C)_{c}$-condition replacing the $(P S)_{c}-$ condition, and it turns out that Lemma 2.3 still holds true with the $(C)_{c}$-condition instead of the $(P S)_{c}-$ condition.

\section{Proof of theorems}

From $(L),\left(W_{1}\right)$ and $\left(W_{2}\right)$, we know that there exists a positive constant $d_{0}$ such that $L(t)+2 d_{0} I_{N} \geq I_{N}$ for all $t \in \mathbb{R}$. Let $\bar{L}(t)=L(t)+2 d_{0} I_{N}$ and $\bar{W}(t, x)=W(t, x)+d_{0}|x|^{2}$. Consider the following fractional Hamiltonian system

$$
\left\{\begin{array}{l}
{ }_{t} D_{\infty}^{\alpha}\left({ }_{-\infty} D_{t}^{\alpha} u\right)(t)+\bar{L}(t) u(t)=\nabla \bar{W}(t, u(t)), t \in \mathbb{R} \\
u \in H^{\alpha}\left(\mathbb{R}, \mathbb{R}^{N}\right)
\end{array}\right.
$$

then $(\overline{\mathscr{F} \mathscr{H} \mathscr{S}})$ is equivalent to $(\mathscr{F} \mathscr{H} \mathscr{S})$. Moreover, it is easy to check that the hypotheses $\left(W_{1}\right)-\left(W_{5}\right)$ still hold for $\bar{W}$ provided that those hold for $W$, and $\bar{L}$ satisfies the conditions $\left(L_{0}\right),\left(L_{2}\right)$. Hence, in what follows, we always assume without loss of generality that $L$ satisfies $\left(L_{0}\right)$ instead of $(L)$.

Consider the variational functional $\Phi$ associated to $(\mathscr{F} \mathscr{H} \mathscr{S})$ :

$$
\Phi(u)=\frac{1}{2} \int_{\mathbb{R}}\left[\left.\left.\right|_{-\infty} D_{t}^{\alpha} u(t)\right|^{2}+L(t) u(t) \cdot u(t)\right] d t-\int_{\mathbb{R}} W(t, u) d t
$$

defined on the space $X^{\alpha}$ introduced in Section 2. In the following, to simplify the notation, we will note the norm $\|\cdot\|_{X^{\alpha}}$ of $X^{\alpha}$ by $\|\cdot\|$.

Lemma 3.1. Under assumptions $\left(L_{0}\right),\left(L_{2}\right)$ and $\left(W_{1}\right)$, the functional

$$
\psi(u)=\int_{\mathbb{R}} W(t, u) d t
$$

is continuously differentiable on $\mathrm{X}^{\alpha}$ and

$$
\psi^{\prime}(u) v=\int_{\mathbb{R}} \nabla W(t, u) \cdot v d t, \forall u, v \in X^{\alpha} .
$$


Proof. By $\left(W_{1}\right)$, there exist constants $a_{0}, R_{0}>0$ such that

$$
|\nabla W(t, x)| \leq a_{0}|x|, \forall(t, x) \in \mathbb{R} \times \mathbb{R}^{N} \text { with }|x| \leq R_{0} .
$$

For any given $u \in X^{\alpha}$, we know that $u \in H^{\alpha}\left(\mathbb{R}, \mathbb{R}^{N}\right)$ and hence there exists a constant $T_{0}>0$ such that

$$
|u(t)| \leq \frac{R_{0}}{2}, \forall|t| \geq T_{0}
$$

By (2.3), for any $v \in X^{\alpha}$ with $\|v\| \leq \frac{R_{0}}{2 \eta_{\infty}}$, we have

$$
\|v\|_{L^{\infty}} \leq \frac{R_{0}}{2}
$$

Combining (3.2)-(3.4) and $\left(W_{1}\right)$, by the Mean Value Theorem and Hölder's inequality, for any $T \geq T_{0}$ and $v \in X^{\alpha}$ with $\|v\| \leq \frac{R_{0}}{2 \eta_{\infty}}$, one has

$$
\begin{aligned}
\left|\int_{|t| \geq T}[W(t, u+v)-W(t, u)-\nabla W(t, u) \cdot v] d t\right| & =\left|\int_{|t| \geq T} \int_{0}^{1}[\nabla W(t, u+s v)-\nabla W(t, u)] . v d s d t\right| \\
& \leq 2 a_{0} \int_{|t| \geq T}(|u|+|v|)|v| d t \leq 2 a_{0}\left(\int_{|t| \geq T}(|u|+|v|)^{2} d t\right)^{\frac{1}{2}}\|v\|_{L^{2}} \\
& \leq 2 a_{0} \eta_{2}\left[\left(\int_{|t| \geq T}|u|^{2} d t\right)^{\frac{1}{2}}+\eta_{2}\|v\|\right]\|v\| .
\end{aligned}
$$

Since $u \in L^{2}(\mathbb{R})$, for any $\varepsilon>0$, there exist $0<\alpha_{1}<\frac{R_{0}}{2 \eta_{\infty}}$ and $T_{\varepsilon} \geq T_{0}$ such that for all $v \in X^{\alpha}$ with $\|v\| \leq \alpha_{1}$

$$
2 a_{0} \eta_{2}\left[\left(\int_{|t| \geq T_{\varepsilon}}|u|^{2} d t\right)^{\frac{1}{2}}+\eta_{2}\|v\|\right] \leq \frac{\varepsilon}{2}
$$

It is well known that the functional

$$
\psi_{\varepsilon}(u)=\int_{\left[-T_{\varepsilon}, T_{\varepsilon}\right]} W(t, u) d t
$$

is continuously differentiable on $H^{1}\left(\left[-T_{\varepsilon}, T_{\varepsilon}\right], \mathbb{R}^{N}\right)$. Thus, since $X^{\alpha}$ is compactly embedded in $H^{\alpha}(\mathbb{R})$, there exists a constant $\alpha_{2}>0$ such that for all $\|v\| \leq \alpha_{2}$

$$
\left|\int_{\left[-R_{\varepsilon}, R_{\varepsilon}\right]}[W(t, u+v)-W(t, u)-\nabla W(t, u) \cdot v] d t\right| \leq \frac{\varepsilon}{2}\|v\| .
$$

Taking $\alpha=\min \left\{\alpha_{1}, \alpha_{2}\right\}$, then (3.5)-(3.7) imply

$$
\left|\int_{\mathbb{R}}[W(t, u+v)-W(t, u)-\nabla W(t, u) \cdot v] d t\right| \leq \varepsilon\|v\|
$$

for all $v \in X^{\alpha}$ with $\|v\| \leq \alpha$. Therefore, $\psi$ is differentiable on $X^{\alpha}$ and satisfies (3.1).

It remains to prove that $\psi^{\prime}$ is continuous. Let $u_{n} \longrightarrow u$ in $X^{\alpha}$. By Hölder's inequality, we have

$$
\begin{aligned}
\left\|\psi^{\prime}\left(u_{n}\right)-\psi^{\prime}(u)\right\|_{E^{\prime}} & =\sup _{\|v\|=1}\left|\psi^{\prime}\left(u_{n}\right) v-\psi^{\prime}(u) v\right| \\
& =\sup _{\|v\|=1}\left|\int_{\mathbb{R}}\left[\nabla W\left(t, u_{n}\right)-\nabla W(t, u)\right] \cdot v d t\right| \\
& \leq \sup _{\|v\|=1}\left(\int_{\mathbb{R}}\left|\nabla W\left(t, u_{n}\right)-\nabla W(t, u)\right|^{2} d t\right)^{\frac{1}{2}}\|v\|_{L^{2}} \\
& \leq \eta_{2}\left(\int_{\mathbb{R}}\left|\nabla W\left(t, u_{n}\right)-\nabla W(t, u)\right|^{2} d t\right)^{\frac{1}{2}} .
\end{aligned}
$$

Lemma 2.3 implies that $u_{n} \longrightarrow u$ in $L^{2}(\mathbb{R})$. Let $M$ be a positive constant such that $\left\|u_{n}\right\|_{L^{2}} \leq M$ for all integer $n$. By $\left(W_{1}\right)$, for any $\varepsilon>0$, there exists a constant $0<r<R_{0}$ such that for all $t \in \mathbb{R}$ and $|x| \leq r$

$$
|\nabla W(t, x)| \leq \frac{\varepsilon}{2\left(M+\|u\|_{L^{2}}\right)}|x|
$$

Due to (3.9) and the facts that $u \in H^{\alpha}(\mathbb{R})$ and $u_{n} \longrightarrow u$ in $L^{\infty}(\mathbb{R})$, there exists $R_{\varepsilon}>R_{0}$ and $N_{1} \in \mathbb{N}$ such that for all $|t| \geq R_{\varepsilon}$ and $n \geq N_{1}$

$$
\left|\nabla W\left(t, u_{n}(t)\right)\right| \leq \frac{\varepsilon}{2\left(M+\|u\|_{L^{2}}\right)}\left|u_{n}(t)\right|
$$

and

$$
|\nabla W(t, u(t))| \leq \frac{\varepsilon}{2\left(M+\|u\|_{L^{2}}\right)}|u(t)| .
$$


Thus

$$
\begin{aligned}
\left(\int_{|t| \geq T_{\varepsilon}}\left|\nabla W\left(t, u_{n}\right)-\nabla W(t, u)\right|^{2} d t\right)^{\frac{1}{2}} & \leq\left(\int_{|t| \geq T_{\varepsilon}}\left|\nabla W\left(t, u_{n}\right)\right|^{2} d t\right)^{\frac{1}{2}}+\left(\int_{|t| \geq T_{\varepsilon}}|\nabla W(t, u)|^{2} d t\right)^{\frac{1}{2}} \\
& \leq \frac{\varepsilon}{2\left(M+\|u\|_{L^{2}}\right)}\left(\left\|u_{n}\right\|_{L^{2}}+\|u\|_{L^{2}}\right) \\
& \leq \frac{\varepsilon}{2} .
\end{aligned}
$$

Observing that $u_{n} \longrightarrow u$ in $L^{\infty}(\mathbb{R})$, then by Lebesgue's Dominated Convergence Theorem, we have

$$
\left(\int_{\left[-T_{\varepsilon}, T_{\varepsilon}\right]}\left|\nabla W\left(t, u_{n}\right)-\nabla W(t, u)\right|^{2} d t\right)^{\frac{1}{2}} \longrightarrow 0 \text { as } n \longrightarrow \infty .
$$

Hence, there is $N_{2} \in \mathbb{N}$ such that for all $n \geq N_{2}$

$$
\left(\int_{\left[-T_{\varepsilon}, T_{\varepsilon}\right]}\left|\nabla W\left(t, u_{n}\right)-\nabla W(t, u)\right|^{2} d t\right)^{\frac{1}{2}} \leq \frac{\varepsilon}{2},
$$

which together with (3.10) implies that for all $n \geq \max \left\{N_{1}, N_{2}\right\}$

$$
\left(\int_{\mathbb{R}}\left|\nabla W\left(t, u_{n}\right)-\nabla W(t, u)\right|^{2} d t\right)^{\frac{1}{2}} \leq \varepsilon .
$$

Combining this with (3.8) implies that $\psi^{\prime}\left(u_{n}\right) \longrightarrow \psi^{\prime}(u)$ as $n \longrightarrow \infty$ and then $\psi \in C^{1}\left(X^{\alpha}, \mathbb{R}\right)$. The proof of Lemma 3.1 is completed.

From Lemma 3.1, we deduce that $\Phi \in C^{1}\left(X^{\alpha}, \mathbb{R}\right)$ and

$$
\Phi^{\prime}(u) v=<u, v>-\int_{\mathbb{R}} \nabla W(t, u) \cdot v d t, \forall u, v \in X^{\alpha} .
$$

Moreover, if $u \in X^{\alpha}$ is a critical point of $\Phi$, we have

$$
{ }_{t} D_{\infty}^{\alpha}\left({ }_{-\infty} D_{t}^{\alpha} u\right)(t)=-L(t) u(t)+\nabla W(t, u(t))
$$

which implies that $u$ is a solution of $(\mathscr{F} \mathscr{H} \mathscr{S})$.

Lemma 3.2. Under assumptions $\left(L_{0}\right),\left(L_{2}\right),\left(W_{1}\right)$ and $\left(W_{2}\right)$, for any finite dimensional subspace $\widetilde{E} \subset X^{\alpha}$, there is $R=R(\widetilde{E})>0$ such that

$$
\Phi(u) \leq 0, \forall u \in \widetilde{E},\|u\| \geq R .
$$

Proof. We will prove the following

$$
\Phi(u) \longrightarrow-\infty \text { as }\|u\| \longrightarrow \infty, u \in \widetilde{E} .
$$

Arguing indirectly, assume that there exists a sequence $\left(u_{n}\right) \subset \widetilde{E}$ with $\left\|u_{n}\right\| \longrightarrow \infty$ and a constant $M>0$ such that $\Phi\left(u_{n}\right) \geq-M$ for all $n \in \mathbb{N}$. Set $v_{n}=\frac{u_{n}}{\left\|u_{n}\right\|}$, then $\left\|v_{n}\right\|=1$. Passing to a subsequence if necessary, we may assume that $v_{n} \rightarrow v$ in $X^{\alpha}$. Since $\widetilde{E}$ is finite dimensional, we have $v_{n} \longrightarrow v$ in $\widetilde{E}$ and $v_{n} \longrightarrow v$ a.e. on $\mathbb{R}$. It follows that $\|v\|=1$. For $0 \leq a<b$, let

$$
\begin{aligned}
& \Omega_{n}(a, b)=\left\{t \in \mathbb{R} / a \leq\left|u_{n}(t)\right|<b\right\} \\
& A=\{t \in \mathbb{R} / v(t) \neq 0\} .
\end{aligned}
$$

Since $v \neq 0$, then meas $(A)>0$. For a.e. $t \in \mathbb{R}$, we have $\lim _{n \longrightarrow \infty}\left|u_{n}(t)\right|=\infty$, hence $t \in \Omega_{n}\left(R_{0}, \infty\right)$ for $n$ large enough. Since $v_{n}(t) \longrightarrow v(t)$ a.e. $t \in \mathbb{R}$, we have $\chi_{\Omega_{n}\left(R_{0}, \infty\right)}(t)\left|v_{n}(t)\right| \longrightarrow|v(t)|$ a.e. $t \in A$, where $\chi_{\Omega}$ denotes the characteristic function of $\Omega$. Hence, it follows from $\left(W_{1}\right)$, $\left(W_{2}\right)$ and Fatou's Lemma that

$$
\begin{aligned}
0 & =\lim _{n \longrightarrow \infty} \frac{-M}{\left\|u_{n}\right\|^{2}} \leq \lim _{n \longrightarrow \infty} \frac{\Phi\left(u_{n}\right)}{\left\|u_{n}\right\|^{2}}=\lim _{n \longrightarrow \infty}\left[\frac{1}{2}-\int_{\mathbb{R}} \frac{W\left(t, u_{n}\right)\left|v_{n}\right|^{2}}{\left|u_{n}\right|^{2}} d t\right] \\
& =\lim _{n \longrightarrow \infty}\left[\frac{1}{2}-\int_{\Omega_{n}\left(0, R_{0}\right)} \frac{W\left(t, u_{n}\right)\left|v_{n}\right|^{2}}{\left|u_{n}\right|^{2}} d t-\int_{\Omega_{n}\left(R_{0}, \infty\right)} \frac{W\left(t, u_{n}\right)\left|v_{n}\right|^{2}}{\left|u_{n}\right|^{2}} d t\right] \\
& \leq \limsup _{n \longrightarrow \infty}\left[\frac{1}{2}+\frac{a_{0}}{2} \int_{\mathbb{R}}\left|v_{n}\right|^{2} d t-\int_{\Omega_{n}\left(R_{0}, \infty\right)} \frac{W\left(t, u_{n}\right)\left|v_{n}\right|^{2}}{\left|u_{n}\right|^{2}} d t\right] \\
& \leq \frac{1}{2}+\frac{a_{0}}{2} \eta_{2}^{2}-\liminf _{n \longrightarrow \infty} \int_{\Omega_{n}\left(R_{0}, \infty\right)} \frac{W\left(t, u_{n}\right)\left|v_{n}\right|^{2}}{\left|u_{n}\right|^{2}} d t \\
& \leq \frac{1}{2}+\frac{a_{0}}{2} \eta_{2}^{2}-\int_{\mathbb{R}} \liminf _{n \longrightarrow \infty} \frac{W\left(t, u_{n}\right)\left|v_{n}\right|^{2}}{\left|u_{n}\right|^{2}} \chi_{\mid \Omega_{n}\left(R_{0}, \infty\right)}(t) d t=-\infty
\end{aligned}
$$

which is a contradiction. Hence (3.13) and then (3.12) is verified. The proof of Lemma 3.2 is completed. 
Let $\left(e_{j}\right)_{j \in \mathbb{N}}$ be an orthonormal basis of $X^{\alpha}$ and define $X_{j}=\mathbb{R} e_{j}$

$$
Y_{k}=\oplus_{j=1}^{k} X_{j}, Z_{k}=\overline{\oplus_{j=k+1}^{\infty} X_{j}}, k \in \mathbb{N} .
$$

Lemma 3.3. Suppose $\left(L_{0}\right)$ and $\left(L_{2}\right)$ hold. Then for any $p \in[2, \infty]$

$$
l_{p}(k)=\sup _{u \in Z_{k},\|u\|=1}\|u\|_{L^{p}} \longrightarrow 0 \text { as } k \longrightarrow \infty
$$

Proof. It is clear that $0<l_{p}(k+1) \leq l_{p}(k)$, so that $l_{p}(k) \longrightarrow \bar{l}_{p}$ as $k \longrightarrow \infty$. For every $k \geq 1$, there exists $u_{k} \in Z_{k}$ such that $\left\|u_{k}\right\|=1$ and $\left\|u_{k}\right\|_{L^{p}}>\frac{1}{2} l_{p}(k)$. For any $v \in X^{\alpha}$, let $v=\sum_{i=1}^{\infty} v_{i} e_{i}$. By the Cauchy-Schwartz inequality, one has

$$
\begin{aligned}
\left|<u_{k}, v>\right| & =\left|<u_{k}, \sum_{i=1}^{\infty} v_{i} e_{i}>\right|=\left|<u_{k}, \sum_{i=k+1}^{\infty} v_{i} e_{i}>\right| \\
& \leq\left\|u_{k}\right\|\left\|\sum_{i=k+1}^{\infty} v_{i} e_{i}\left|\leq \sum_{i=k+1}^{\infty}\right| v_{i} \mid\right\| e_{i} \| \longrightarrow 0 \text { as } k \longrightarrow \infty
\end{aligned}
$$

which implies that $u_{k} \rightarrow 0$. Without loss of generality, Lemma 2.3 implies that $u_{k} \longrightarrow 0$ in $L^{2}(\mathbb{R})$. Thus we have proved that $\bar{l}_{p}=0$. The proof of Lemma 3.3 is completed.

By (3.15), we can choose an integer $m \geq 1$ such that

$$
\|u\|_{L^{2}} \leq \frac{1}{2 a_{0}}\|u\|, \forall u \in Z_{m}
$$

In the following, we will apply Lemma 3.1 with $Y=Y_{m}$ and $Z=Z_{m}$.

Lemma 3.4. Under assumptions $\left(L_{0}\right),\left(L_{2}\right)$ and $\left(W_{1}\right)$, there exist constants $\rho, \alpha>0$ such that $\Phi_{\mid \partial B_{\rho} \cap Z} \geq \alpha$.

Proof. If $\|u\|=\frac{R_{0}}{\eta_{\infty}}$, then by (2.3), we have $\|u\|_{L^{\infty}} \leq R_{0}$. Hence, it follows from $\left(W_{1}\right)$ that

$$
W(t, u(t)) \leq \frac{a_{0}}{2}|u(t)|^{2}, \forall u \in X^{\alpha},\|u\|=\frac{R_{0}}{\eta_{\infty}} .
$$

Combining (3.16) with (3.17) yields for all $u \in Z$ with $\|u\|=\frac{R_{0}}{\eta_{\infty}}=\rho$

$$
\begin{aligned}
\Phi(u)=\frac{1}{2}\|u\|^{2}-\int_{\mathbb{R}} W(t, u) d t & \geq \frac{1}{2}\|u\|^{2}-\frac{a_{0}}{2} \int_{\mathbb{R}}|u|^{2} d t \geq \frac{1}{2}\|u\|^{2}-\frac{a_{0}}{2}\|u\|_{L^{2}}^{2} \\
& \geq \frac{1}{4}\|u\|^{2}=\frac{1}{4}\left(\frac{R_{0}}{\eta_{\infty}}\right)^{2}=\alpha .
\end{aligned}
$$

The proof of Lemma 3.4 is completed.

Proof of Theorem 1.1 By assumptions $\left(W_{1}\right)$ and $\left(W_{3}\right)$, it is clear that

$$
\Phi(0)=0 \text { and } \Phi(-u)=\Phi(u), \forall u \in X^{\alpha} .
$$

Thus the condition (a) of Lemma 3.1 is satisfied. Lemmas 3.2, 3.4 imply that conditions (b) and (c) of Lemma 3.1 are satisfied. It remains to prove that $\Phi$ satisfies the $(C)_{c}$-condition.

Lemma 3.5. Assume that $\left(L_{0}\right),\left(L_{2}\right),\left(W_{1}\right),\left(W_{2}\right)$ and $\left(W_{4}\right)$ are satisfied. Then $\Phi$ verifies the $(C)_{c}-$ condition for all $c \in \mathbb{R}$.

Proof. Let $\left(u_{n}\right)$ be a $(C)_{c}$ sequence, that is

$$
\Phi\left(u_{n}\right) \longrightarrow \text { and }\left\|\Phi^{\prime}\left(u_{n}\right)\right\|\left(1+\left\|u_{n}\right\|\right) \longrightarrow 0 \text { as } n \longrightarrow \infty .
$$

Firstly, we prove that $\left(u_{n}\right)$ is bounded in $X^{\alpha}$. Arguing by contradiction, suppose that $\left\|u_{n}\right\| \longrightarrow \infty$ as $n \longrightarrow \infty$. Let $v_{n}=\frac{u_{n}}{\left\|u_{n}\right\|}$. Then $\left\|v_{n}\right\|=1$. Observe that for $n$ large enough

$$
c+1 \geq \Phi\left(u_{n}\right)-\frac{1}{2} \Phi^{\prime}\left(u_{n}\right) u_{n}=\int_{\mathbb{R}} \widehat{W}\left(t, u_{n}\right) d t .
$$

It follows from (3.21) that

$$
\frac{1}{2} \leq \lim \sup _{n \rightarrow \infty} \int_{\mathbb{R}} \frac{\left|W\left(t, u_{n}\right)\right|}{\left\|u_{n}\right\|^{2}} d t .
$$


Passing to a subsequence if necessary, we may assume that $v_{n} \rightarrow v$ in $X^{\alpha}$. Then by Lemma 2.3, without loss of generality, we have $v_{n} \longrightarrow v$ in $L^{2}(\mathbb{R})$ and $v_{n} \longrightarrow v$ a.e. on $\mathbb{R}$.

If $v=0$, then $v_{n} \longrightarrow 0$ in $L^{2}(\mathbb{R})$ and $v_{n} \longrightarrow 0$ a.e. on $\mathbb{R}$. Hence, it follows from $\left(W_{1}\right)$ that

$$
\int_{\Omega_{n}\left(0, R_{0}\right)} \frac{\left|W\left(t, u_{n}\right)\right|}{\left\|u_{n}\right\|^{2}} d t=\int_{\Omega_{n}\left(0, R_{0}\right)} \frac{\left|W\left(t, u_{n}\right)\right|}{\left|u_{n}\right|^{2}}\left|v_{n}\right|^{2} d t \leq \frac{a_{0}}{2} \int_{\Omega_{n}\left(0, R_{0}\right)}\left|v_{n}\right|^{2} d t \leq \frac{a_{0}}{2}\left\|v_{n}\right\|_{L^{2}}^{2} \longrightarrow 0 \text { as } n \longrightarrow \infty .
$$

From $\left(W_{4}\right)$ and (3.22), one has

$$
\begin{aligned}
\int_{\Omega_{n}\left(R_{0}, \infty\right)} \frac{\left|W\left(t, u_{n}\right)\right|}{\left\|u_{n}\right\|^{2}} d t & =\int_{\Omega_{n}\left(R_{0}, \infty\right)} \frac{\left|W\left(t, u_{n}\right)\right|}{\left|u_{n}\right|^{2}}\left|v_{n}\right|^{2} d t \\
& \leq \frac{c_{0}\left\|v_{n}\right\|_{L^{\infty}}^{2-v}}{\left\|u_{n}\right\|^{v}} \int_{\Omega_{n}\left(R_{0}, \infty\right)} \widehat{W}\left(t, u_{n}\right) d t \\
& \leq \frac{c_{0}\left\|v_{n}\right\|_{L^{\infty}}^{2-v}}{\left\|u_{n}\right\|^{v}}\left[1+c-\int_{\Omega_{n}\left(0, R_{0}\right)} \widehat{W}\left(t, u_{n}\right) d t\right] \\
& \leq \frac{c_{0} \eta_{\infty}^{2-v}}{\left\|u_{n}\right\|^{v}}\left[1+c-\int_{\Omega_{n}\left(0, R_{0}\right)} g(t) d t\right] \\
& \leq \frac{c_{0} \eta_{\infty}^{2-v}}{\left\|u_{n}\right\|^{v}}\left[1+c+\int_{\mathbb{R}}|g(t)| d t\right] \longrightarrow 0 \text { as } n \longrightarrow \infty
\end{aligned}
$$

Combining (3.22) with (3.23) yields

$$
\int_{\mathbb{R}} \frac{\left|W\left(t, u_{n}\right)\right|}{\left\|u_{n}\right\|^{2}} d t=\int_{\Omega_{n}\left(0, R_{0}\right)} \frac{\left|W\left(t, u_{n}\right)\right|}{\left\|u_{n}\right\|^{2}} d t+\int_{\Omega_{n}\left(R_{0}, \infty\right)} \frac{\left|W\left(t, u_{n}\right)\right|}{\left\|u_{n}\right\|^{2}} d t \longrightarrow 0 \text { as } n \longrightarrow \infty
$$

which contradicts (3.21).

If $v \neq 0$. By a similar fashion as for (3.14), we can get a contradiction. Therefore, $\left(u_{n}\right)$ is bounded in $X^{\alpha}$.

Next, we prove that $\left(u_{n}\right)$ possesses a convergent subsequence. Without loss of generality, we can assume by Remark 2.4 that $u_{n} \longrightarrow u$ in $L^{2}(\mathbb{R})$. Using Hölder's inequality, $\left(W_{1}\right)$ and the fact that $\nabla W$ is bounde'd in $\mathbb{R} \times B_{r}(0)$ for all $r>0$, we can show that

$$
\int_{\mathbb{R}}\left[\nabla W\left(t, u_{n}\right)-\nabla W(t, u)\right] \cdot\left(u_{n}-u\right) d t \longrightarrow 0 \text { as } n \longrightarrow \infty .
$$

Observe that

$$
\left\|u_{n}-u\right\|^{2}=\left(\Phi^{\prime}\left(u_{n}\right)-\Phi^{\prime}(u)\right)\left(u_{n}-u\right)+\int_{\mathbb{R}}\left[\nabla W\left(t, u_{n}\right)-\nabla W(t, u)\right] \cdot\left(u_{n}-u\right) d t .
$$

It is clear that

$$
\left(\Phi^{\prime}\left(u_{n}\right)-\Phi^{\prime}(u)\right)\left(u_{n}-u\right) \longrightarrow 0 \text { as } n \longrightarrow \infty .
$$

Combining (3.24) - (3.26), we get $\left\|u_{n}-u\right\| \longrightarrow 0$ as $n \longrightarrow \infty$. The proof of Lemma 3.5 is completed.

Consequently, Lemma 2.3 together with Remark 2.2 imply that $\Phi$ possesses an unbounded sequence of critical points. Therefore $(\mathscr{F} \mathscr{H} \mathscr{S})$ possesses infinitely many solutions. The proof of Theorem 1.1 is completed.

\section{Proof of Theorem 1.2}

Lemma 3.6. Under assumptions $\left(L_{0}\right),\left(L_{2}\right),\left(W_{1}\right)-\left(W_{3}\right)$ and $\left(W_{5}\right)$, $\Phi$ satisfies the $(C)_{c}-$ condition for all $c \in \mathbb{R}$.

Proof. Let $c \in \mathbb{R}$ and $\left(u_{n}\right) \subset X^{\alpha}$ satisfying (3.19). First, we prove that $\left(u_{n}\right)$ is bounded in $X^{\alpha}$. Arguing by contradiction, suppose that $\left\|u_{n}\right\| \longrightarrow \infty$ as $n \longrightarrow \infty$. Let $v_{n}=\frac{u_{n}}{\left\|u_{n}\right\|}$. Then $\left\|v_{n}\right\|=1$ and $\left\|v_{n}\right\| \leq \eta_{p}\left\|v_{n}\right\|_{L^{p}}$ for $2 \leq p \leq \infty$. By $\left(W_{5}\right)$, one has for $n$ large enough

$$
\begin{aligned}
c+1 \geq \Phi\left(u_{n}\right)-\frac{1}{\mu} \Phi^{\prime}\left(u_{n}\right) u_{n} & =\frac{\mu-2}{2 \mu}\left\|u_{n}\right\|^{2}+\int_{\mathbb{R}}\left[\frac{1}{\mu} \nabla W\left(t, u_{n}\right) \cdot u_{n}-W\left(t, u_{n}\right)\right] d t \\
& \geq \frac{\mu-2}{2 \mu}\left\|u_{n}\right\|^{2}-\frac{\rho_{0}}{\mu}\left\|u_{n}\right\|_{L^{2}}^{2}
\end{aligned}
$$

which implies

$$
\frac{\mu-2}{2 \rho_{0}} \leq \lim \sup _{n \longrightarrow \infty}\left\|v_{n}\right\|_{L^{2}}^{2} .
$$

Taking a subsequence if necessary, we may assume that $v_{n} \longrightarrow v$ in $L^{2}(\mathbb{R})$ and $v_{n} \longrightarrow v$ a.e. on $\mathbb{R}$. Hence, it follows from (3.27) that $v \neq 0$. By a similar fashion as for (3.14), we can get a contradiction. Therefore $\left(u_{n}\right)$ is bounded in $X^{\alpha}$. The rest of the proof is the same as that in Lemma 3.5 and the proof of Lemma 3.6 is completed.

We conclude as in the proof of Theorem 1.1 that $\Phi$ possesses an unbounded sequence of critical points and the proof of Theorem 1.2 is completed. 


\section{Conclusion}

Using the variational methods and critical point theory, we proved that the fractional Hamiltonian system $(\mathscr{F} \mathscr{H} \mathscr{S})$ possesses infinitely many nontrivial solutions, where $L$ is neither uniformly positive definite nor coercive and $W$ does not satisfy the classical superquadratic growth conditions like the well-known Ambrosetti-Rabinowitz superquadratic condition. Recent results in the literature are generalized and significantly improved.

\section{Acknowledgement}

The author thanks the referee for valuable suggestions.

\section{References}

[1] O. Agrawal, J. Tenreiro Machado, J. Sabatier, Fractional derivatives and their applications, Springer-Verlag, Berlin, 2004

[2] T. Bartolo, V. Benci, D. Fortunato, Abstract critical point theorems and applications to some nonlinear problems with strong resonance at infinity, Nonlinear Analysis, Vol. 7 , No. 9 (1983) 981-1012;

[3] N. Nyamoradi, A. Alsaedi, B. Ahmad, Y. Zou, Multiplicity of homoclinic solutions for fractional Hamiltonian systems with subquadratic potential, Entropy 2017, 19,50,1-24;

[4] N. Nyamoradi, A. Alsaedi, B. Ahmad, Y. Zou, Variational approach to homoclinic solutions for fractional Hamiltonian systems, J. Optim. Theory Appl. 2017;

[5] Z. Bai, H. Lü, Positive solutions for boundary value problem of nonlinear fractional differential equation, J. Math. Anal. Appl. (2005), 311, 495-505;

[6] Z. Bai, Y. Zhang, The existence of solutions for a fractional multi-point boundary value problem, Computers and Mathematics with Applications 2010, 69, 2364-2372;

[7] P. Chen, X. He, X.H. Tang, Infinitely many solutions for a class of Hamiltonian systems via critical point theory, Math. Meth. Appl. Sci. 2016, 39, 1005-1019;

[8] Y. Li, B. Dai, Existence and multiplicity of nontrivial solutions for Liouville-Weyl fractional nonlinear Schrödinger equation, RA SAM (2017);

[9] W. Jiang, The existence of solutions for boundary value problems of fractional differential equations at resonance, Nonlinear Analysis (2011), 74, 1987-1994;

[10] F. Jiao, Y. Zhou, Existence results for fractional boundary value problem via critical point theory, Intern. Journal of Bif. and Chaos, 22, No. 4 (2012), $1-17$

[11] R. Hiffer, Applications of fractional calculus in physics, World Science, Singapore, 2000;

[12] S. G. Samko, A.A Kilbas, O.I. Marichev, Fractional integrals and derivatives, Theory and applications, Gordon and Breach, Switzerland 1993;

[13] A.A. Kilbas, H.M. Srivastawa, J.J. Trujillo, Theory and applications of fractional differential equations, North-Holland Mathematical Studies; Vol. 204, Singapore 2006;

[14] S. Liang, J. Zhang, Positive solutions for boundary value problems of nonlinear fractional differential equations, Nonlinear Analysis, 2009, 71, $5545-5550$;

[15] J. Mawhin, M. Willem, Critical point theory and Hamiltonian systems, Applied Mathematical Sciences, Springer, Berlin, 1989;

[16] A. Mèndez, C. Torres, Multiplicity of solutions for fractional Hamiltonian systems with Liouville-Weyl fractional derivative, arXiv: 1409.0765v1[mathph] 2 Sep. 2014;

[17] K. Miller, B. Ross, An introduction to differential equations, Wiley and Sons, New York, 1993;

[18] I. Pollubny, Fractional differential equations, Academic Press, 1999;

[19] P.H. Rabinowitz, Minimax methods in critical point theory with applications to differential equations, CBMS Reg. Conf. Ser. in Math., Vol. 65, American Mathematical Society, Providence, RI, 1986;

[20] K. Tang, Multiple homoclinic solutions for a class of fractional Hamiltonian systems, Progr. Fract. DIff. Appl. 2, , No. 4 (2016), 265-276;

[21] C. Torres, Existence of solutions for fractional Hamiltonian systems, Electr. J. DIff. Eq., Vol. 2013 (2013), No. 259, 1-12;

[22] C. Torres Ledesma, Existence of solutions for fractional Hamiltonian systems with nonlinear derivative dependence in $\mathbb{R}, \mathrm{J}$. Fractional Calculus and Applications; Vol. 7 (2) (2016) 74-87;

[23] X. Wu, Z. Zhang, Solutions for perturbed fractional Hamiltonian systems without coercive conditions, Boundary Value Problems (2015) 2015: 149, $1-12$;

[24] S. Zhang, Existence of solutions for the fractional equations with nonlinear boundary conditions, Computers and Mathematics with Applications (2011), 61, 1202-1208;

[25] S. Zhang, Existence of solutions for a boundary value problems of fractional differential equations at resonance, Nonlinear Analysis (2011): 74, 1987-1994.

[26] Z. Zhang, R. Yuan, Existence of solutions to fractional Hamiltonian systems with combined nonlinearities, Electr. J. Diff. Eq., Vol. 2016 (2016) No. 40, $1-13$;

[27] Z. Zhang, R. Yuan, Solutions for subquadratic fractional Hamiltonian systems without coercive conditions, Math. Meth. Appl. Sci. (2014) 37, 2934-2945;

[28] Z. Zhang, R. Yuan, Variational approach to solutions for a class of fractional Hamiltonian systems, Math. Meth. Appl. Sci. (2014) 37, 1873-1883; 